\title{
DHAV-1 Inhibits Type I Interferon Signaling to Assist Viral Adaption by Increasing the Expression of SOCS3
}

\author{
Jinyan Xie ${ }^{1,2 \dagger}$, Mingshu Wang ${ }^{1,2,3 \dagger}$, Anchun Cheng ${ }^{1,2,3 *}$, Xin-Xin Zhao ${ }^{1,2,3}$, Mafeng Liu ${ }^{1,2,3}$, \\ Dekang Zhu ${ }^{2,3}$, Shun Chen ${ }^{1,2,3}$, Renyong Jia ${ }^{1,2,3}$, Qiao Yang ${ }^{1,2,3}$, Ying $\mathrm{Wu}^{1,2,3}$, \\ Shaqiu Zhang ${ }^{1,2,3}$, Yunya Liu ${ }^{1,2,3}$, Yanling $\mathrm{Yu}^{1,2,3}$, Ling Zhang ${ }^{1,2,3}$ and Xiaoyue Chen ${ }^{1,2,3}$ \\ 1 Institute of Preventive Veterinary Medicine, Sichuan Agricultural University, Chengdu, China, ${ }^{2}$ Key Laboratory of Animal \\ Disease and Human Health of Sichuan Province, Sichuan Agricultural University, Chengdu, China, ${ }^{3}$ Avian Disease Research \\ Center, College of Veterinary Medicine, Sichuan Agricultural University, Chengdu, China
}

OPEN ACCESS

Edited by:

Constantinos Petrovas, Vaccine Research Center (NIAID), United States

Reviewed by: Irene Ramos, Icahn School of Medicine at Mount

Sinai, United States Arik Cooper, National Institutes of Health (NIH), United States

*Correspondence: Anchun Cheng

chenganchun@vip.163.com

†These authors have contributed equally to this work as first authors

Specialty section: This article was submitted to Viral Immunology, a section of the journal

Frontiers in Immunology

Received: 12 November 2018 Accepted: 19 March 2019

Published: 09 April 2019

Citation:

Xie J, Wang $M$, Cheng A, Zhao X-X, Liu M, Zhu D, Chen S, Jia R, Yang $Q$, Wu Y, Zhang S, Liu Y, Yu Y, Zhang L and Chen $X$ (2019) DHAV-1 Inhibits Type I Interferon Signaling to Assist

Viral Adaption by Increasing the Expression of SOCS3.

Front. Immunol. 10:731.

doi: 10.3389/fimmu.2019.00731
Duck hepatitis A virus type 1 (DHAV-1) is one of the most lethal pathogens in the duck industry. The attenuated vaccine (the $\mathrm{CH} 60$ strain) is cultivated through serial passage in chicken embryos and is widely used for the prevention and control of the disease. However, the specific mechanism underlying its adaptation in chicken embryos has not been fully elucidated. In this study, we first infected chicken embryo fibroblasts (CEFs) with the DHAV-1 CH60 strain. The peak of viral proliferation occurred within 36-48 $\mathrm{h}$ post-infection. The different DHAV-1 strains significantly induced the expression of IFN $\alpha$, IFN $\gamma$, and Suppressor of cytokine signaling 3 (SOCS3) in CEFs, and we found that SOCS3 overexpression significantly promoted viral replication. Furthermore, SOCS3 overexpression significantly inhibited the expression of IFN $\alpha$ but promoted the expression of IFN $\gamma$. In addition, SOCS3 overexpression clearly decreased the mRNA levels of STAT1 and STAT3 in the Janus kinase (JAK)-STAT signaling pathway and inhibited the expression of the antiviral proteins MX1 and OASL. Immune-precipitation assays indicated that SOCS3 and IFN $\alpha$ do not physically interact. Subcellular localization of SOCS3 and IFN $\alpha$ revealed that SOCS3 was mainly located in the nucleus and cytoplasm, while IFN $\alpha$ was located only in the cytoplasm. Co-localization of these two proteins was not observed in the cytoplasm. In conclusion, the DHAV-1 CH60 strain may inhibit the expression of IFN $\alpha$ by increasing the SOCS3 protein and SOCS3 can in turn decrease STAT1 and STAT3 mRNA levels, thereby inhibiting the antiviral protein MX1 and ultimately promoting viral proliferation, indirectly assisting in viral adaptation in chicken embryos.

\section{Keywords: DHAV-1, IFNs, SOCS3, STATs, MX1}

\section{INTRODUCTION}

Duck hepatitis A virus type 1 (DHAV-1) is one of the most lethal pathogens for ducks, especially ducklings $<1$ week old, as it can cause $100 \%$ morbidity and $95 \%$ mortality (1). DHAV-1 is responsible for acute hepatitis characterized by petechial and ecchymotic hemorrhages of liver surfaces $(2-5)$. To prevent and control the disease, we have established a variety of methods for 
virological detection of DHAV-1 and have characterized the functions of several viral proteins (6-11). At present, the prevention and control of the disease mainly depends on live attenuated vaccines $(12,13)$, such as the DHAV-1 CH60 strain (14). However, the focus and research on attenuated vaccines remain insufficient. The $\mathrm{CH} 60$ strain attenuated vaccine is cultivated through serial passage in chicken embryos and is widely used for the prevention and control of the disease. Intensive research has found that incompatible host translational selection pressure is one of the main mechanisms of viral attenuation and adaptation in chicken embryos (15). In addition, transcriptome sequencing has revealed that infection of chicken embryo livers with the CH60 strain is associated with enhanced type I and II interferon responses, activated innate immune responses, and abundant expression of cytokine signaling molecules 1 and 3 (SOCS1 and SOCS3) (16). Some structural and functional information about genes involved in host interactions with DHAV-1 has been reported recently (17-23). However, the specific mechanisms of viral attenuation and adaptation in chicken embryos remain to be further studied.

The SOCS/CIS family consists of CIS (cytokine-induced SH2containing protein) and SOCS1 to SOCS7, each of which has a central SH2 domain, an amino-terminal domain of variable length and sequence, and a carboxy-terminal 40-amino-acid sequence known as the SOCS box (24). SOCS proteins regulate cytokine signaling mainly through the JAK-STAT signaling pathway, and SOCS1 and SOCS3 play an important role in the development of inflammation and tumors (25). The
SOCS3 usually inhibits the activation of STAT3 via binding to both the JAK kinase and the cytokine receptor. Moreover, SOCS3 also plays a role in mediating the ubiquitination and subsequent proteasome degradation of cytokine/growth factor/hormone receptor (26). Previous studies have revealed that the CH60 strain significantly enhances SOCS1 and SOCS3 mRNA levels in the liver, activates the JAK-STAT signaling pathway and induces type I and II interferon responses (16). However, the role of SOCS proteins in viral attenuation and adaptation in chicken embryos remains unclear. Therefore, this study mainly explored whether the attenuated vaccine inhibits the JAK-STAT signaling pathway by overexpressing the SOCS3 protein, thereby inhibiting the expression of interferons and ultimately promoting its own replication to achieve adaptation.

\section{MATERIALS AND METHODS}

\section{Virus and Cells}

The attenuated DHAV-1 CH60 strain and DHAV-1 WT strain were provided by the Instituted of Preventive Veterinary Medicine, Sichuan Agricultural University. Chicken embryo fibroblasts (CEFs) were cultured in minimum essential medium (MEM) supplemented with $10 \%$ newborn calf serum (Gibco) and incubated at $37^{\circ} \mathrm{C}$ with $5 \% \mathrm{CO}_{2}$ in an incubator. The virus titer was measured as the $50 \%$ tissue culture infective dose $\left(\mathrm{TCID}_{50}\right)$ according to previously described methods.

TABLE 1 | Primers used in this study.

\begin{tabular}{|c|c|c|}
\hline Primer & Forward $\left(5^{\prime}-3^{\prime}\right)$ & Reverse $\left(5^{\prime}-3^{\prime}\right)$ \\
\hline gga-IFN $\alpha-q P C R$ & TCGCAACCTTCACCTCACC & CGCAGGCGCTGTAATCGT \\
\hline gga-IFN $\beta$-qPCR & TCCAGCTCCTTCAGAATACG & TGCGGTCAATCCAGTGTT \\
\hline gga-IFN $\gamma$-qPCR & TCATACTGAGCCAGAvTTGT & AAGTCGTTCATCGGGAGC \\
\hline $\begin{array}{l}\text { gga-SOCS3- } \\
\text { qPCR }\end{array}$ & CGGCACTTCTCACCCTCAG & CAGCTTCAGCACGCAGTCG \\
\hline gga- $\beta$-actin-qPCR & CACAGATCATGTTTGAGACCTT & CATCACAATACCAGTGGTACG \\
\hline $\begin{array}{l}\text { PCAGGS-IFN } \alpha- \\
\text { FLAG }\end{array}$ & $\begin{array}{l}\text { CATCATTTGGCAAAGAATTCACCGCCACCATGGCTGTGCCTGCAA } \\
\text { GCCCA }\end{array}$ & $\begin{array}{l}\text { TTGGCAGAGGGAAAAAGATCTCTACTTATCGTCGTCATCCTTGTAA } \\
\text { TCAGTGCGCGTGTTGCCTG }\end{array}$ \\
\hline $\begin{array}{l}\text { PCAGGS-IFN } \beta- \\
\text { FLAG }\end{array}$ & $\begin{array}{l}\text { CATCATTITGGCAAAGAATTCACCGCCACCATGGCCACTGCAAAC } \\
\text { CATCAGTC }\end{array}$ & $\begin{array}{l}\text { TTGGCAGAGGGAAAAAGATCTTCACTTATCGTCGTCATCCTTGTA } \\
\text { ATCCTGGGTGTTGAGACGTIT }\end{array}$ \\
\hline $\begin{array}{l}\text { PCAGGS-IFN } \gamma- \\
\text { FLAG }\end{array}$ & $\begin{array}{l}\text { CATCATTITGGCAAAGAATTCACCGCCACCATGGCCACTTGCCAG } \\
\text { ACTTACAA }\end{array}$ & $\begin{array}{l}\text { TTGGCAGAGGGAAAAAGATCTTACTTATCGTCGTCATCCTTGTA } \\
\text { ATCGCAATTGCATCTCCTCT }\end{array}$ \\
\hline DHAV-1 F1 & ССТTAATTCAACGTCTAGCCCAC & TGCAAATCAGTTTCAAGGAGTTCT \\
\hline DHAV-1 F2 & CCCTATGCCATCTTGGATCT & СTTCCTGATTGAGTCCACAT \\
\hline DHAV-1 F3 & AATGTCCCAATACAAGGTGA & СССССАAАAATAAAATTTGAA \\
\hline DHAV-1 F4 & AGTCAGCATTAAATGGTGAAGT & CATATACCAAGAGGTTCAGGACG \\
\hline DHAV-1 F5 & CTTCAGTGGCTCCAGGA & TGATCTTCCAAACCAACCA \\
\hline DHAV-1 F6 & CTTGGATTCTTGGTATAGGAAC & СССАТСАССАТТСТАТАAGC \\
\hline DHAV-1 F7 & ATGGCTAAGAAAGCATCT & GTAGGGTAGGGAATAGTAAAG \\
\hline
\end{tabular}




\section{RNA Isolation and CDNA Preparation}

Total RNA was isolated using RNAiso Plus Reagent (TaKaRa, Dalian, China) according to the manufacturer's instructions. Genomic DNA was then removed and reverse transcription was performed using a PrimeScript ${ }^{\mathrm{TM}}$ RT Reagent Kit (Perfect Real Time, TaKaRa) according to the manufacturer's instructions.

\section{Viral RNA Load and Cytokine Expression in CEFs}

The number of viral copies in total RNA was measured using methods previously established in our laboratory (7). Eight genes (IFN $\alpha$, IFN $\beta$, IFN $\gamma$, SOCS3, STAT1, STAT3, MX1, and OASL) and a housekeeping gene ( $\beta$-actin) were analyzed by qPCR using primers designed with Primer Premier 5 (Table 1). The expression levels of immune-related genes were determined by qPCR using a SYBR ${ }^{\circledR}$ Premix Ex Taq ${ }^{\text {TM }}$ II (Tli RNaseH Plus) Kit (TaKaRa) and an Applied CFX96 Real-Time PCR Detection System (Bio-Rad, Hercules, CA, USA). Amplification was performed in $10 \mu \mathrm{l}$ reaction volumes containing $0.5 \mu \mathrm{l}$ of each primer and $1 \mu \mathrm{l}$ of cDNA. The following thermal cycling conditions were used: initial activation at $95^{\circ} \mathrm{C}$ for $30 \mathrm{~s}, 40$ cycles of denaturation at $95^{\circ} \mathrm{C}$ for $5 \mathrm{~s}$ and annealing and extension at $58.6^{\circ} \mathrm{C}$ for $30 \mathrm{~s}$, and a dissociation curve analysis step.

\section{Plasmids}

To construct pCAGGS-IFN $\alpha$-FLAG, pCAGGS-IFN $\beta$-FLAG, pCAGGS-IFN $\gamma$-FLAG, and pCAGGS-SOCS3-His plasmids, IFN $\alpha$ (GenBank: NC_006127.5), IFN $\beta$ (GenBank: NC_006127), IFN $\gamma$ (GenBank: NC_006088), and SOCS3 (GenBank:
NC_006105.5) sequence were amplified from cDNA with PCR and primers (Table 1) and were integrated into the pCAGGS vector with a one-step cloning kit (Vazyme).

To compare the differential sequences of the DHAV-1 CH60 and DHAV-1 CH60 adapted CEF strains, we designed related primers according to the conserved sequence of DHAV1 (F1-F7, Table 1). Amplification was performed in $30 \mu \mathrm{l}$ reaction volumes containing $15 \mu \mathrm{l}$ of PrimeSTAR Max DNA Polymerase (TaKaRa), $1.5 \mu \mathrm{l}$ of each primer and $1 \mu \mathrm{l}$ of cDNA. The following thermal cycling conditions were used: initial activation at $98^{\circ} \mathrm{C}$ for $3 \mathrm{~min}, 30$ cycles of denaturation at $98^{\circ} \mathrm{C}$ for $10 \mathrm{~s}$ and annealing and extension at $55^{\circ} \mathrm{C}$ for $60 \mathrm{~s}$. Fragment 1 and Fragment 7 were integrated into the $\mathrm{pMD}^{\mathrm{TM}} 19-\mathrm{T}$ vector and then sequenced, and Fragment 2-6 were directly sequenced.

To prepare exogenous IFN $\alpha$, CEFs were transfected with pCAGGS-IFN $\alpha$-FLAG plasmids using Lipofectamine 3000 (Invitrogen) according to the manufacturer's instructions. At $48 \mathrm{~h}$ after transfection, cells were harvested and repeatedly frozen and thawed three times, then detected the concentration of IFN $\alpha$ by using chicken IFN- $\alpha$ ELISA kit (TW, shanghai). The concentration of recombinant IFN $\alpha$ was $100 \mathrm{pg} / \mathrm{ml}$.

\section{Western Blot Analysis}

CEFs were plated into 24-well cell culture dishes and cultured overnight. The cells were then transfected with the recombinant plasmids using Lipofectamine 3000 (Invitrogen) according to the manufacturer's instructions. At 36 and $48 \mathrm{~h}$ after transfection, cells were harvested and combined with $5 \times$

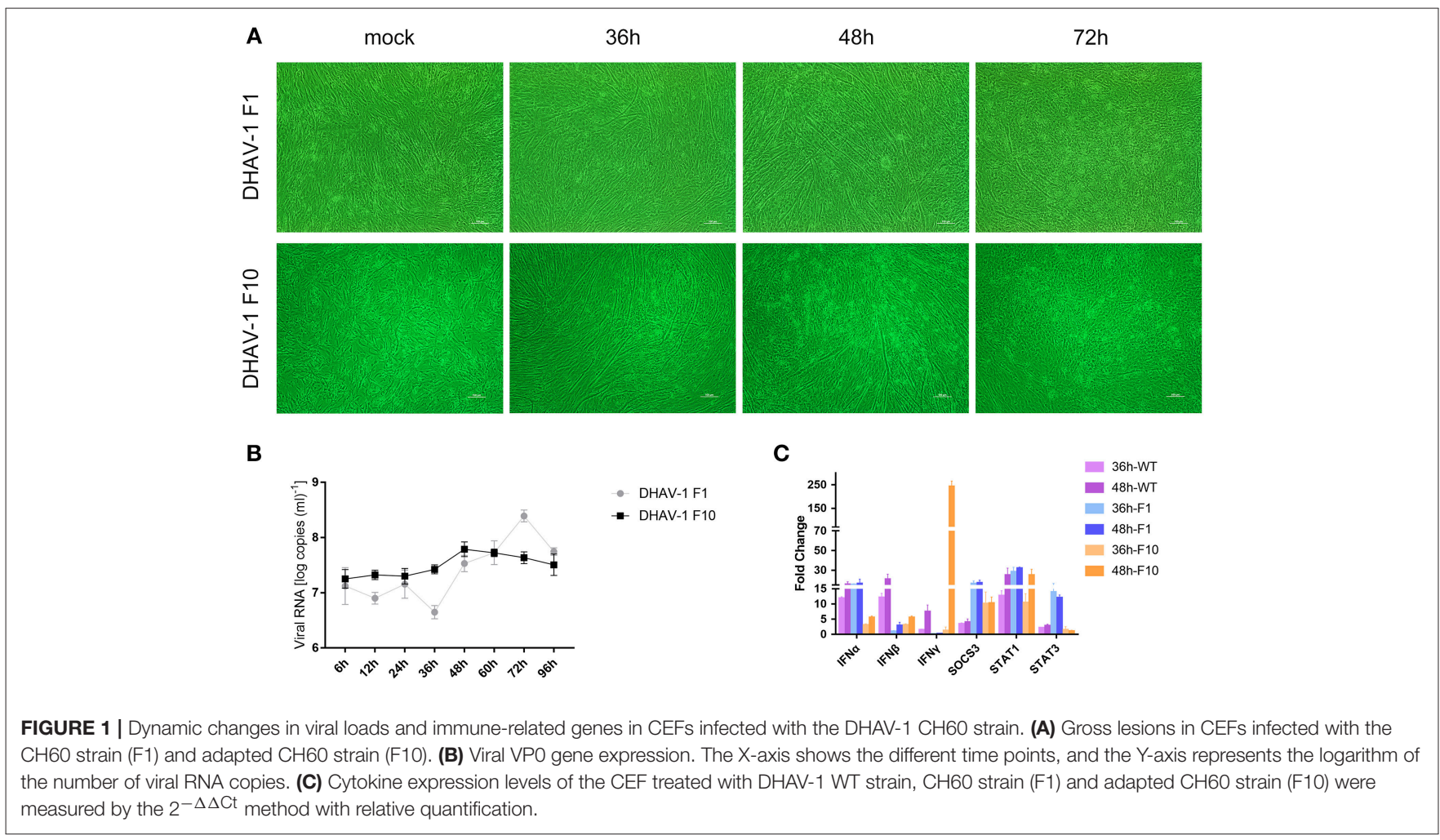


sodium dodecyl sulfate (SDS) loading buffer and then resolved by SDS-polyacrylamide gel electrophoresis (PAGE). The proteins were then transferred onto polyvinylidene fluoride (PVDF) membranes. The membranes were blocked for $4 \mathrm{~h}$ with 5\% non-fat milk/Tris-buffered saline with Tween-20 (TBST) at room temperature and then incubated with a primary antibody against FLAG (Proteintech Group, Wuhan, China), His (Abcam, Cambridge, UK) or STAT1 (CTS) for $12-24 \mathrm{~h}$ at $4^{\circ} \mathrm{C}$. The membranes were rinsed (three times) with TBST and then incubated with a secondary antibody. The protein bands were detected using Western BLoT Chemiluminescence HRP Substrate (TaKaRa). Co-immunoprecipitation was performed as previously described (27).

\section{Indirect Immunofluorescence}

Cells were transfected with the recombinant plasmids using Lipofectamine 3000 (Invitrogen) according to the manufacturer's instructions. At $48 \mathrm{~h}$ after transfection, the cells were rinsed (three times) with phosphate-buffered saline (PBS) and then fixed in $4 \%$ paraformaldehyde overnight. The cells were permeabilized (0.2\% Triton X-100 for $25 \mathrm{~min}$ ), incubated with blocking solution (5\% bovine serum albumin (BSA) in PBS with Tween 20 (PBST) for $60 \mathrm{~min}$ at $37^{\circ} \mathrm{C}$ ), incubated with a primary antibody against FLAG (Proteintech Group, Wuhan, China) or His (Abcam, Cambridge, UK) and then rinsed with PBST. The cells were then incubated with Texas red-conjugated goat antimouse IgG and fluorescein isothiocyanate (FITC)-conjugated goat anti-rabbit IgG secondary antibodies and finally treated with 4',6-diamidino-2-phenylindole (DAPI). Images were captured using an 80i upright microscope (Nikon) and a SPOT Flex camera (28).

\section{shRNA-Mediated Knockdown of SOCS3}

The pGPU6/GFP/Neo-SOCS3-275/371/663 expression vectors were designed and constructed by GenePharma (Shanghai, China), and the sequences of shRNAs were as follows: SOCS3275 5'-GCTTCTACTGGAGCACGGTGA-3', SOCS3-371

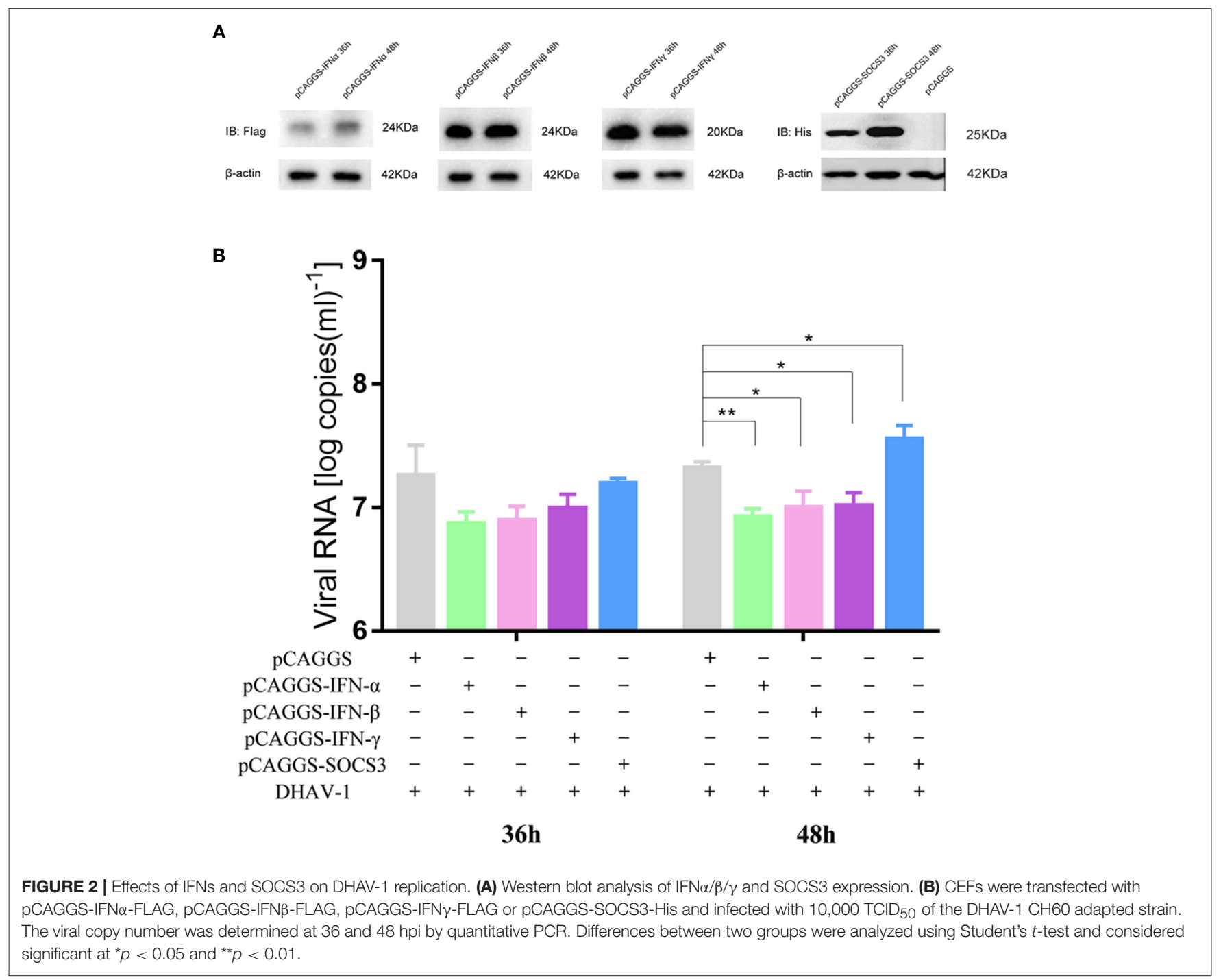


5'-GGCACTTCTTCACCCTCAGCG-3', SOCS3-663 5' GCACCTCTGCCGTAAGACTGT-3'. The CEFs were plated into 24-well cell culture dishes and cultured overnight. The cells were then transfected with the recombinant plasmids using Lipofectamine 3000 (Invitrogen) according to the manufacturer's instructions and infected with the $10^{4}$ TCID $_{50}$ DHAV-1 CH60 adapted strain.

\section{RESULTS}

\section{Replication of DHAV-1 in CEFs}

Previous studies have shown that chicken embryos can be used as an animal model for DHAV-1 (16). However, there is no report of DHAV-1 infection in CEFs. To further explore the interaction between DHAV-1 and the host immune system, the DHAV-1 CH60 strain was adapted to CEFs in 10 serial passages, and viral proliferation and lesions gradually stabilized in CEFs (Figure 1A). To further investigate the replication of DHAV-1 in CEFs, we infected CEFs with 10,000 TCID $_{50}$ DHAV-1 and harvested cells to determine the viral copy number at 6,12 , $24,36,48,60,72$, and $96 \mathrm{~h}$ post-infection (hpi). A significant proliferation of the DHAV-1 CH60 strain occurred from 36 to 72 hpi, a peak was observed at $72 \mathrm{hpi}$, and then a decreasing trend was observed at 96 hpi (Figure 1B, F1). The adapted strain (F10) presented more stable replication than the DHAV-1 CH60 strain, and the peak of proliferation (48 hpi) was earlier than that of the DHAV-1 CH60 strain (72 hpi). However, the peak copy number of the DHAV-1 CH60 strain was higher than that of the adapted strain. Compared with the DHAV-1 CH60 strain, the adapted strain had nine mutations, and the detailed sequence is shown in Supplementary Figure 3.. Previous studies have revealed that DHAV-1 CH60 strain infection significantly induces type I and II interferon responses, activates the JAK-STAT signaling pathway, and enhances SOCS3 mRNA levels in the livers of chicken embryos. We suspected that $\mathrm{CH} 60$ strain infection would also alter this pathway in CEFs. Therefore, we determined the mRNA levels of type I and II interferons, SOCS3 and STAT1/3 at 36 and 48 hpi. The three different DHAV-1 strains significantly induced the expression of IFN $\alpha$, IFN $\beta$, IFN $\gamma$, SOCS3, and STAT1 at 48 hpi. Notably, compared with the WT strain, the expression of type I IFN induced by the adapted strain (F10) decreased and the expression of SOCS3 increased (Figure 1C).
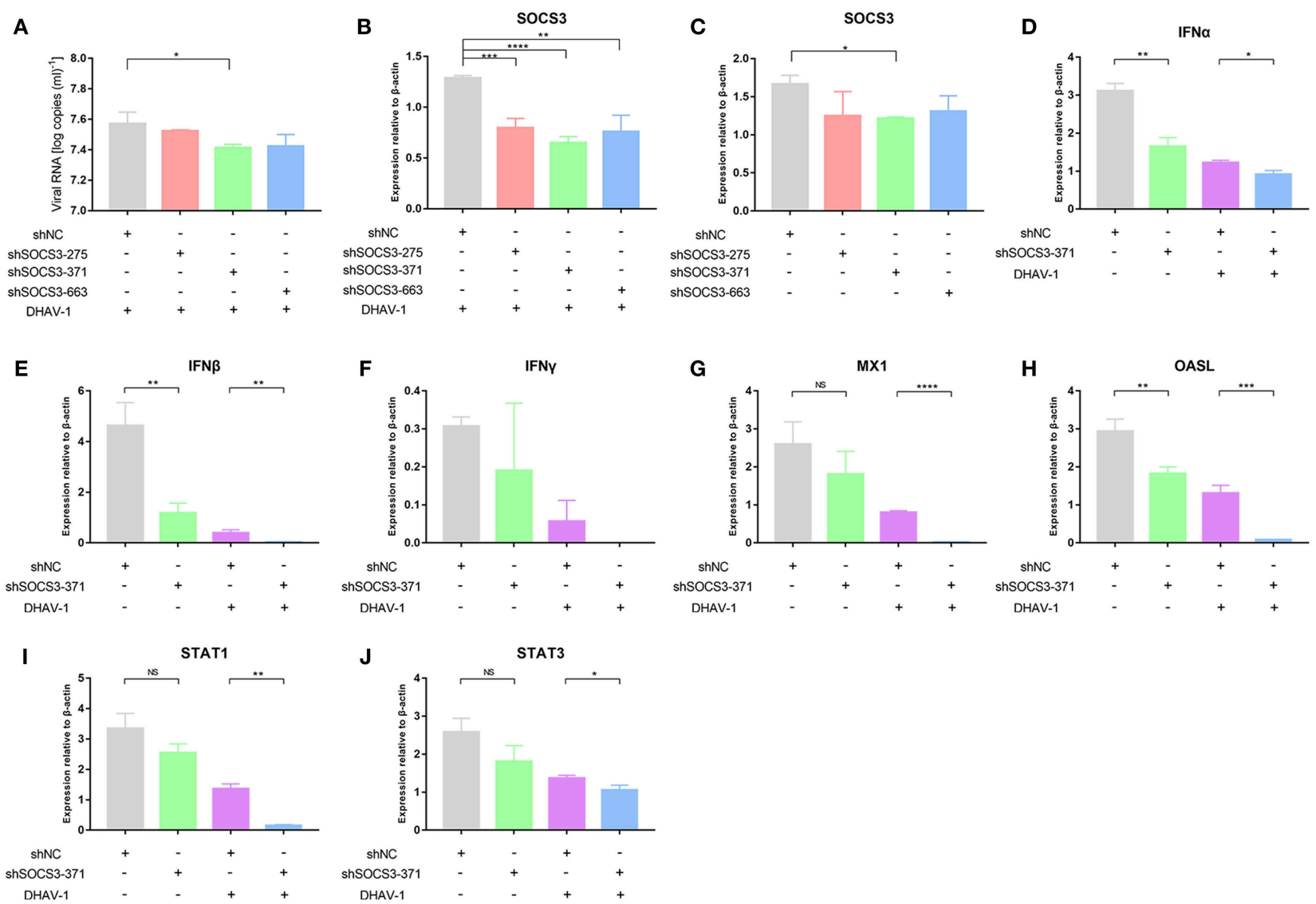

FIGURE 3 | Effects of shSOCS3 on DHAV-1 replication and endogenous interferon pathway components. CEFs were transfected with pGPU6/GFP/Neo-SOCS3 and infected with $10^{4} \mathrm{TCID}_{50}$ of the DHAV-1 CH60 adapted strain. The viral copy number was determined at 24 hpi by quantitative PCR. Quantitative PCR analysis of IFN $\alpha / \beta / \gamma$, SOCS3, MX1, OASL, STAT1 and STAT3 mRNA levels. Differences between two groups were analyzed using Student's $t$-test and considered significant at ${ }^{*} p$ $<0.05,{ }^{* *} p<0.01,{ }^{* * *} p<0.001$, and ${ }^{* * * *} p<0.0001$. 


\section{Effects of IFNs and SOCS3 on DHAV-1 Replication}

We demonstrated that DHAV-1 enhanced IFN and SOCS3 mRNA levels in CEFs. SOCS, a family of intracellular proteins, regulates the responses of immune cells to cytokines (29), while IFNs, as antiviral molecules, play a vital role in the immune system (30); however, the effects of IFNs and SOCS3 on the replication of DHAV-1 are still unknown. Therefore, we constructed four plasmids, including pCAGGS-IFN $\alpha$-FLAG, pCAGGS-IFN $\beta$-FLAG, pCAGGS-IFN $\gamma$-FLAG, and pCAGGSSOCS3-His, that could successfully express in CEFs (Figure 2A). To further explore the effects of IFNs and SOCS3 on DHAV-1 replication, we transfected the above four plasmids into CEFs alone or in CEFs infected with 10,000 TCID $_{50}$ of the DHAV1 CH60 adapted strain and determined the viral copy number at 36 and 48 hpi by quantitative PCR. Compared with that in the empty vector group, DHAV- 1 replication in the IFN $\alpha$, IFN $\beta$, and IFN $\gamma$ groups was decreased at $36 \mathrm{~h}$, although significant inhibition was not observed; in addition, SOCS3 did not show significant changes. IFN $\alpha$, IFN $\beta$, and IFN $\gamma$ also significantly inhibited viral replication at $48 \mathrm{hpi}$, while SOCS3 significantly promoted viral replication (Figure 2B). Then, we designed three shRNAs against SOCS3 (shSOCS3-275/371/663) and found that shSOCS3-371 could significantly interfere with the transcription of SOCS3 (Figure 3C). We also found that viral replication decreased with the use of shSOCS3-371 (Figures 3A,B). Finally, the endogenous levels of the interferon pathway components $($ IFN $\alpha / \beta / \gamma$, MX1, OASL, STAT1 and STAT3) were tested. Except for IFN $\gamma$, shSOCS3 could significantly inhibit the transcription of type I interferon, MX1, OASL, STAT1 and STAT3 (Figure 3).
A

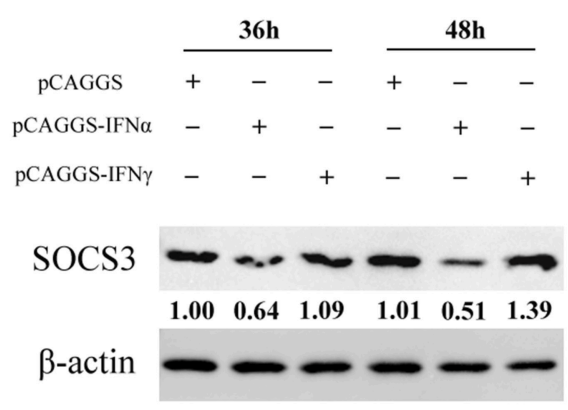

C

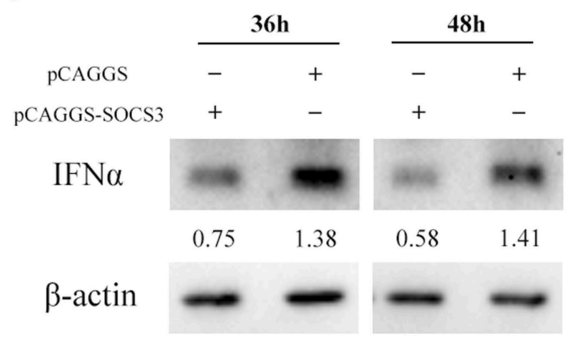

D

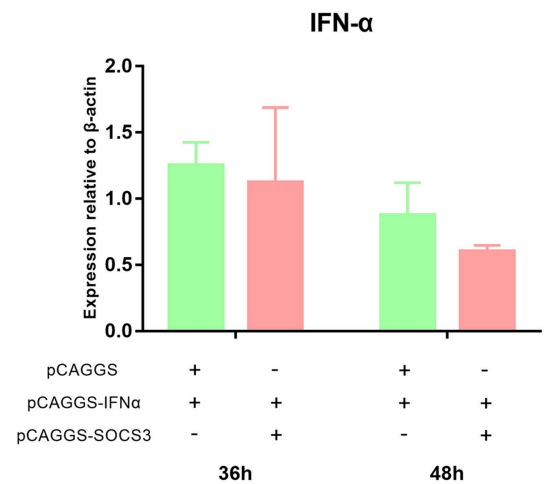

B
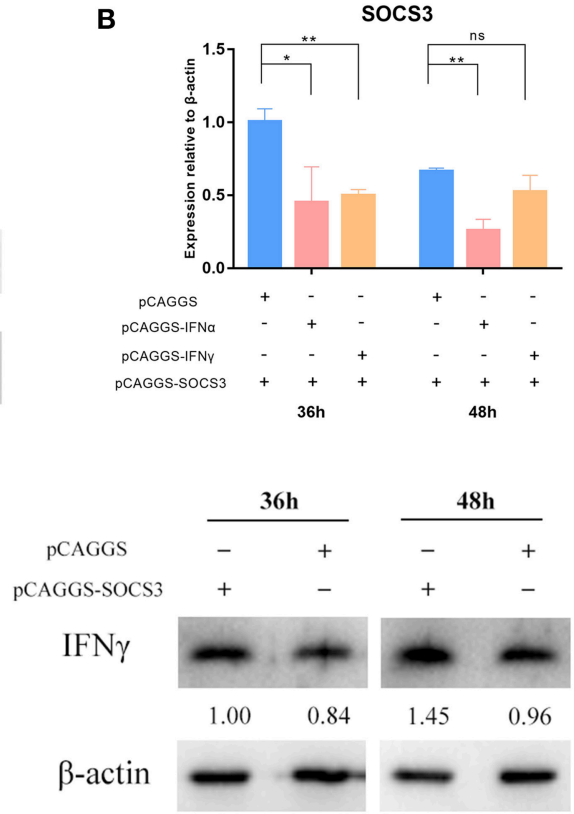

IFN-Y



FIGURE 4 | SOCS3 inhibits the expression of IFN $\alpha$. pCAGGS-SOCS3-His and pCAGGS-IFN $\alpha / \gamma$-FLAG were co-transfected into CEFs, which were harvested 36 and $48 \mathrm{~h}$ after transfection. (A) Western blot analysis of SOCS3 expression. (B) Quantitative PCR analysis of SOCS3 mRNA levels. (C) Western blot analysis of IFN $\alpha / \gamma$ expression. (D) Quantitative PCR analysis of IFN $\alpha / \gamma$ mRNA levels. Differences between two groups were analyzed using Student's $t$-test and considered significant at ${ }^{*} p<0.05$ and ${ }^{* *} p<0.01$. 


\section{SOCS3 Inhibits the Expression of IFN $\alpha$}

We suspected that the virus inhibited the expression of IFNs by hijacking the SOCS3 protein to promote viral replication. Therefore, to further explore how the virus used the SOCS3 protein to promote its own replication, we co-transfected pCAGGS-SOCS3-His and pCAGGS-IFN $\alpha$-FLAG into CEFs and harvested the cells at 36 and $48 \mathrm{~h}$ after transfection. Western blot analysis was used to determine the expression of SOCS3 and IFN $\alpha$. The results showed that IFN $\alpha$ significantly inhibited the expression of SOCS3 (Figure 4A; Supplementary Figure 1), while SOCS3 in turn also significantly inhibited the expression of IFN $\alpha$ (Figure 4C). In addition, SOCS3 and IFN $\alpha$ also inhibited each other at the transcriptional level (Figures 4B,D; Supplementary Figure 2). It is worth noting that we found no mutual inhibition between SOCS3 and IFN $\gamma$. In contrast, each of those proteins significantly promoted the expression of the other at $48 \mathrm{~h}$ after transfection (Figures $4 \mathbf{A}, \mathbf{C}$ ), and SOCS3 also significantly promoted IFN $\gamma$ mRNA expression (Figure 4D).

We confirmed that SOCS3 inhibited the expression of IFN $\alpha$. To further explore the location of their interaction, we used indirect immunofluorescence to detect the subcellular localization of SOCS3 and IFN $\alpha$. The results showed that IFN $\alpha$ is localized in the cytoplasm and is mainly located around the nucleus (Figure 5A), while SOCS3 is mostly localized in the nucleus, although some SOCS3 is also located around the nucleus (Figure 5B). In cells co-transfected with SOCS3 and IFN $\alpha$, SOCS 3 and IFN $\alpha$ were not co-localized in the cytoplasm (Figure 5C). Subsequently, we performed immune-precipitation assays and confirmed that IFN $\alpha$ and SOCS3 do not physically interact (Figure 5D; Supplementary Figure 4), suggesting that the interaction between these two proteins occurs indirectly in the cytoplasm.

\section{SOCS3 Inhibits the Expression of the Antiviral Proteins MX1 and OASL}

The innate immune system recognizes invasive viruses through pattern recognition receptors (PRRs) and then activates downstream pathways to produce IFNs; the IFNs then bind to corresponding receptors to activate the JAK-STAT signaling pathway, ultimately inducing antiviral responses to produce the antiviral proteins MX1, OASL, and others (31). Therefore, we wanted to further explore whether SOCS3 affected the expression of downstream antiviral proteins. Compared with that after IFN $\alpha$ and IFN $\gamma$ transfection, the expression of MX1 and OASL was significantly inhibited after SOCS3 transfection. In addition, the expression of MX1, but not OASL, was significantly inhibited after co-transfection of IFN $\alpha$ and SOCS3 into CEFs compared with that after co-transfection of IFN $\alpha$ and pCAGGS
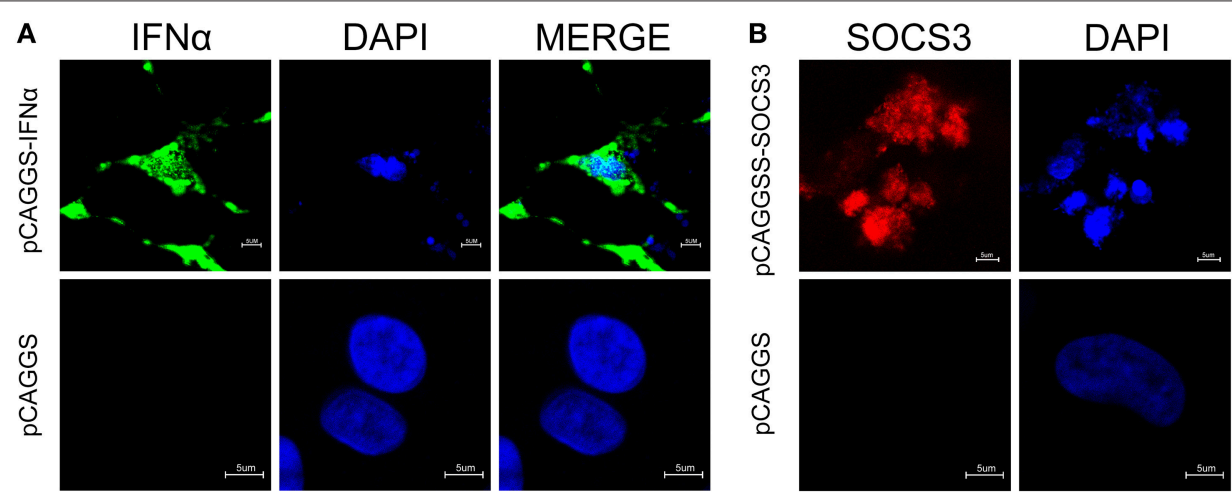

\section{MERGE}
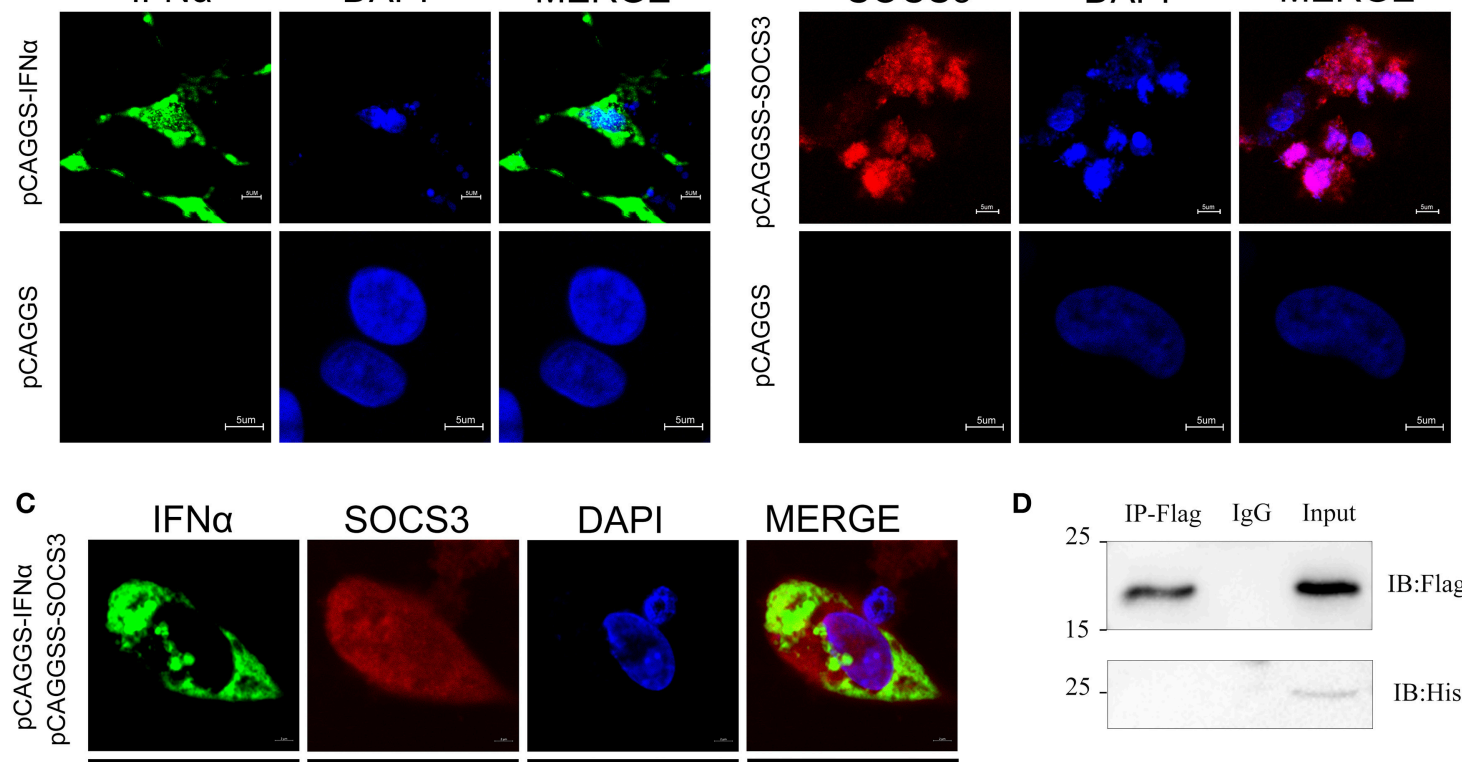

D

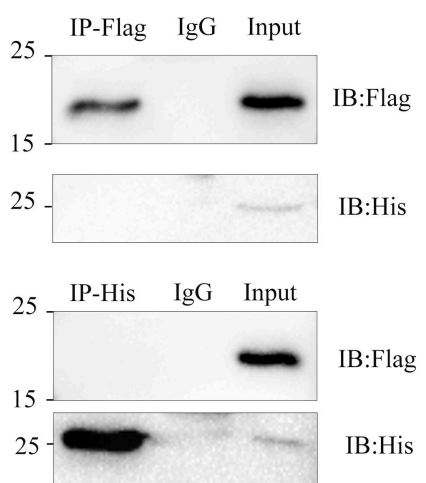

FIGURE 5 | Subcellular localization of SOCS3 and IFN $\alpha$. (A) Localization of IFN $\alpha$ (green) in CEFs. DAPI staining shows the nucleus in blue. (B) Localization of SOCS3 (red) in CEFs. DAPI staining shows the nucleus in blue. (C) Co-localization of IFN $\alpha$ (green) and SOCS3 (red) in CEFs. DAPI staining shows the nucleus in blue. (D) CEFs were transfected with pCAGGS-IFN $\alpha$-Flag and pCAGGS-SOCS3-His for $48 \mathrm{~h}$ before the coimmunoprecipitation and immunoblot analysis with the indicated antibodies. 
(Figure 6A). These findings indicated that SOCS3 indeed inhibits the expression of IFN $\alpha$ and downstream MX1. Furthermore, we wanted to explore whether this process would ultimately promote viral replication. Therefore, we co-transfected SOCS3 and IFN $\alpha$ into CEFs and infected the cells with 10,000 TCID 50 of the DHAV-1 adapted strain. The results showed that the viral copy number in the SOCS3 and IFN $\alpha$-co-transfected group was slightly lower than that in the SOCS3 and pCAGGSco-transfected group; however, the viral copy number was significantly upregulated compared with that in the IFN $\alpha$ and
pCAGGS-co-transfected group (Figure 6B), indicating that SOCS3 inhibited the expression of MX1 and promoted viral replication. However, we found that SOCS3 did not inhibit the expression of IFN $\gamma$ and thereby inhibited the expression of antiviral proteins, ultimately affecting viral replication.

\section{SOCS3 Inhibits the Expression of STAT1 and STAT3}

The JAK-STAT signaling pathway plays an important role in the induction of host cytokines. To further explore whether SOCS3
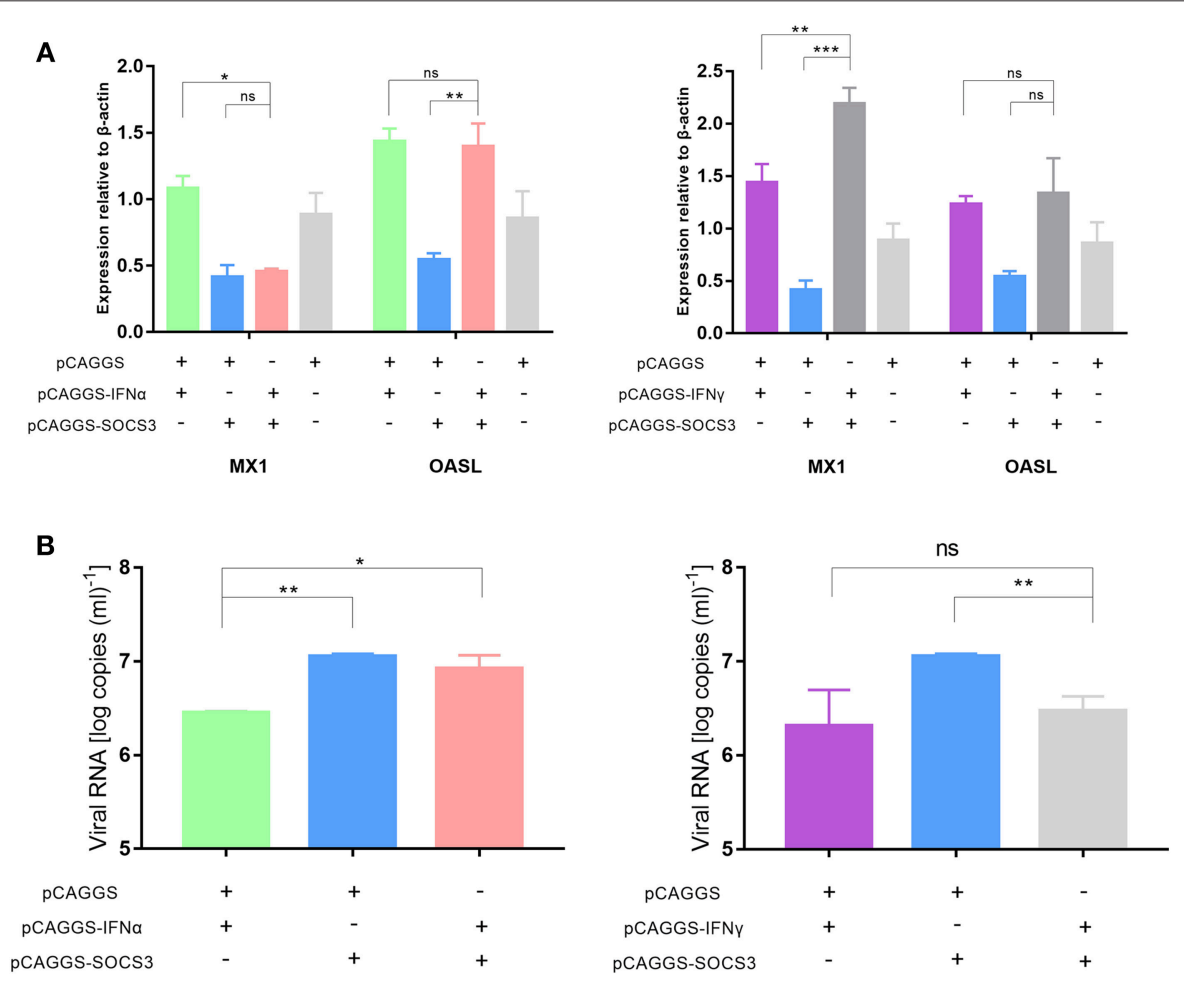

FIGURE 6 | SOCS3 inhibits the expression of the antiviral proteins MX1 and OASL. pCAGGS-SOCS3-His and pCAGGS-IFNa/ $\gamma$-FLAG were co-transfected into CEFs that were infected with 10,000 TCID 50 of the DHAV-1 CH60 adapted strain. The cells were harvested 36 and $48 \mathrm{~h}$ after transfection. (A) Quantitative PCR analysis of the MX1 and OASL mRNA levels. (B) Quantitative PCR analysis of the viral copy number. Differences between two groups were analyzed using Student's $t$-test and considered significant at ${ }^{\star} p<0.05,{ }^{* *} p<0.01$, and ${ }^{* \star *} p<0.0001$.
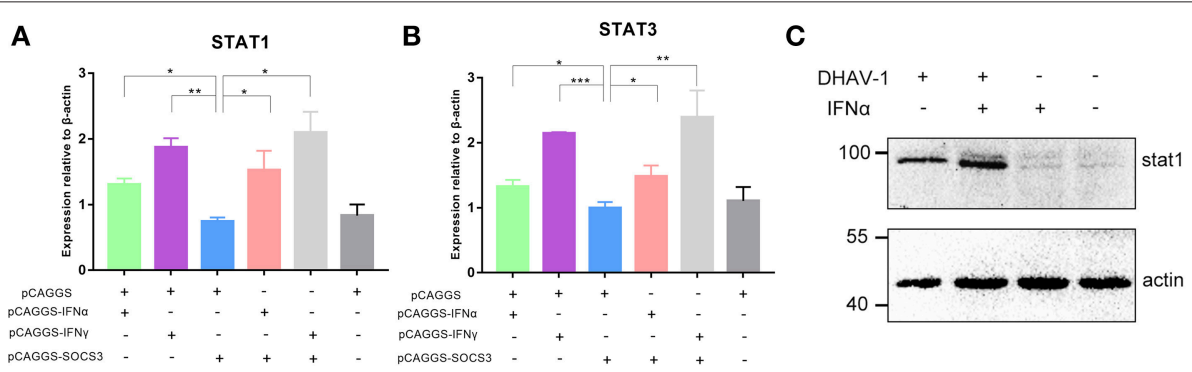

FIGURE 7 | SOCS3 inhibits the expression of STAT1 and STAT3. (A,B) pCAGGS-SOCS3-His and pCAGGS-IFN $\alpha / \gamma$-FLAG were co-transfected into CEFs that were infected with 10,000 TCID 50 of the DHAV-1 CH60 strain. The cells were harvested 36 and $48 \mathrm{~h}$ after transfection. Quantitative PCR was used to analyse the STAT1 and STAT3 mRNA levels. Differences between two groups were analyzed using Student's $t$-test and considered significant at ${ }^{\star} p<0.05$, ${ }^{\star \star} p<0.01$, and ${ }^{\star \star \star} p<$ 0.0001. (C) Western blot analysis of STAT1 expression. DHAV-1 infected CEFs at 48 hpi and after the addition of 100 pg IFN $\alpha$ to stimulate CEFs at $37^{\circ} \mathrm{C}$ for 15 min. Cells were harvested after stimulation. 
inhibited the production of antiviral molecules by affecting the JAK-STAT signaling pathway, we determined the transcript levels of STAT1 and STAT3 and found that SOCS3 significantly inhibited the expression of STAT1 and STAT3 compared to IFN $\alpha / \gamma$, suggesting that SOCS3 can indeed affect the JAKSTAT signaling pathway. However, when IFN $\alpha$ or IFN $\gamma$ was cotransfected with SOCS3, STAT1 and STAT3 were not significantly inhibited, indicating that SOCS3 could not inhibit the expression of STAT1 and STAT3 by directly inhibiting IFN $\alpha$ (Figures 7A,B). Then, we detected the expression of STAT1 and found that DHAV-1 could induce the expression of STAT1, with increased STAT1 levels observed in the infected cells in the presence of IFN $\alpha$ (Figure 7C).

\section{DISCUSSION}

Previous studies have shown that the $\mathrm{CH} 60$ strain attenuated vaccine undergoes different types of mutations during serial passage, such as synonymous and non-synonymous mutation in the coding region and mutation in the UTRs. Conceivably, these mutations drive the adaptation of the virus to the environment (15). The changes in attenuated vaccines and virulent strains in the host before and after adaptation are also worthy of attention. Through transcriptome sequencing, we have found that attenuated vaccines induce type I and II interferon responses, activate innate immune responses, and simultaneously enhance the expression of SOCS1 and SOCS3 (16). We suspected that the adaptation of attenuated vaccines during the process of serial passage is related to high expression of SOCS proteins. Further investigation revealed that overexpression of the SOCS3 protein inhibited the expression of IFN $\alpha$; in addition, SOCS 3 inhibited the expression of the key molecules STAT1 and STAT3 in the downstream JAK-STAT pathway and inhibited the expression of the terminal antiviral protein MX1, ultimately promoting CH60 strain replication and thus indirectly assisting in viral adaptation (Figure 8).

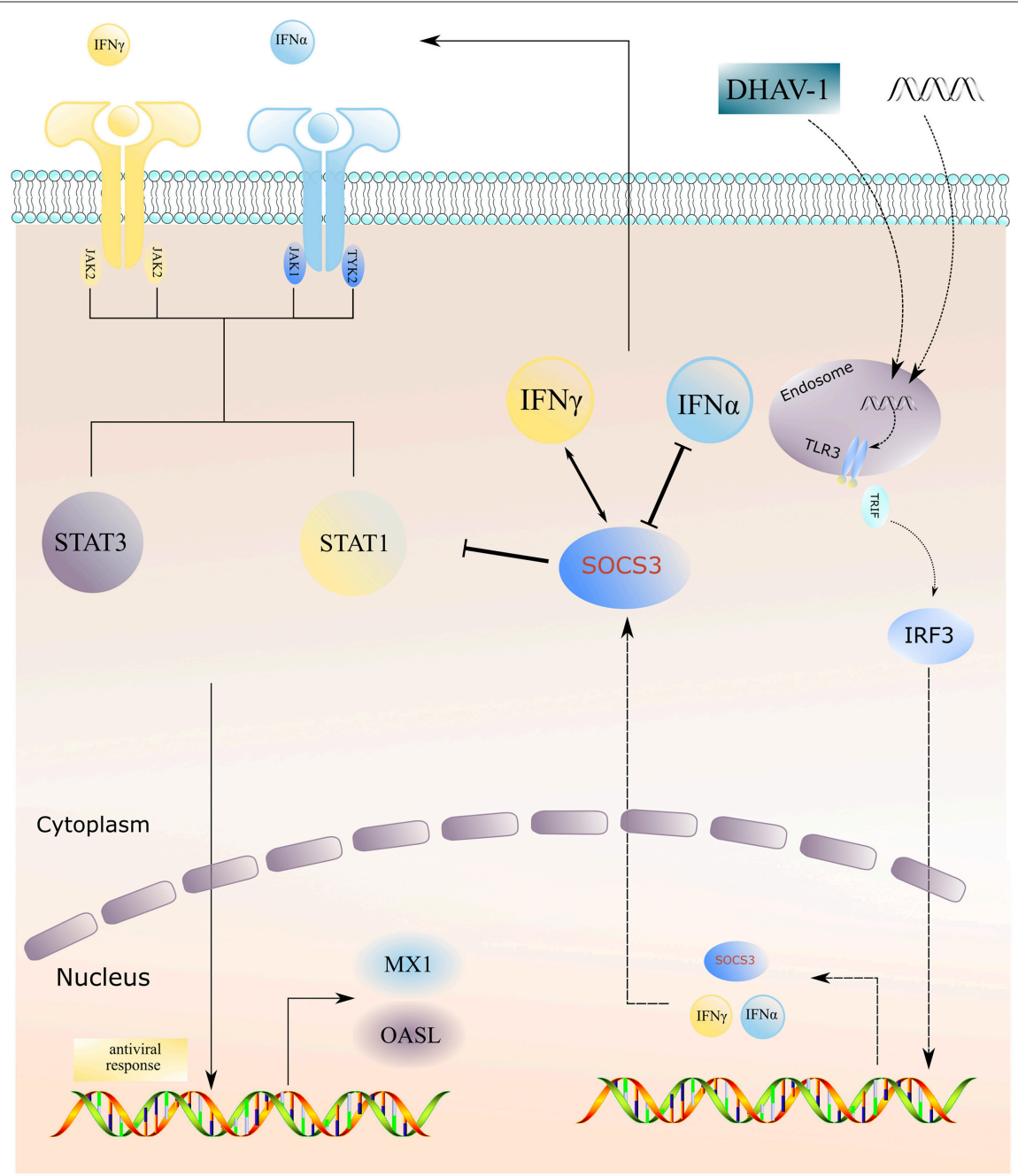

FIGURE 8 | Schematic model of DHAV-1 inhibiting type I interferon signaling by hijacking the SOCS3 protein. Overexpression of the SOCS3 protein inhibits the expression of IFN $\alpha$; in addition, SOCS3 inhibits the expression of the key molecules STAT1 and STAT3 in the downstream JAK-STAT pathway and inhibits the expression of the terminal antiviral protein MX1, ultimately promoting virus replication and indirectly assisting in viral adaptation. 
Innate immunity is the first line of host defense against invading pathogens (32). In innate immunity, invading pathogens are recognized by PRRs, stimulating the downstream signaling pathway to produce type I interferons, which then activate the JAK-STAT signaling pathway; this process ultimately induces the expression of IFN-stimulated genes (ISGs) and leads to the destruction of the pathogens. However, to replicate and survive, attenuated viruses have evolved a complete immune evasion mechanism to achieve their own replication (33). The induction of the SOCS3 protein by attenuated vaccines has drawn our attention, and we have further confirmed that SOCS3 can significantly inhibit the expression of IFN $\alpha$ and the antiviral protein MX1 to promote viral replication, which is consistent with our hypothesis. Another study on HCV similarly found that $\mathrm{HCV}$ evades immune responses by hijacking SOCS function; the $\mathrm{HCV}$ core protein induces SOCS3 expression and the inhibition of STAT1 activation, tyrosine phosphorylation and nuclear localization, ultimately neutralizing the antiviral effects of IFNs (34). Moreover, the data indicate that higher hepatic SOCS3 expression is associated with a poor response to antiviral therapy in human HCV patients (35). However, we found that SOCS3 could not inhibit type II interferon or IFN $\gamma$ but rather had a promoting effect. IFN $\gamma$ bridges innate and adaptive immunity by modulating the differentiation of naive $\mathrm{T}$ cells into either Th1 (cell immunity) or Th2 (humoral immunity) cells, while it directs long-term control of viral infection by inducing cytotoxic immunity through the recognition of cell surface-bound viral antigens complexed with MHC proteins (30). In addition, it has been demonstrated that SOCS1 is a potent inhibitor of the IFN $\gamma$ signaling pathway $(36,37)$ and can prevent atherosclerosis by inhibiting IFN $\gamma$ signaling (38). Therefore, we hypothesize that attenuated vaccines may regulate IFN $\gamma$ signaling through SOCS1 rather than SOCS3, and the specific mechanism needs further investigation.

The JAK-STAT signaling pathway manages more than 50 downstream cytokines and growth factors and is regarded as a central communication node for the immune system. This pathway is mainly composed of Janus kinases (JAKs) and signal transducers and activators of transcription (STATs); JAKs can be subdivided into JAK1, JAK2, JAK3, and TYK2, while STATs can be subdivided into STAT1, STAT2, STAT3, STAT4, STAT5A, STAT5B, and STAT6 (39). A variety of viruses disrupt the IFN $\alpha$ signaling pathway by degrading the major components of the JAK-STAT signaling pathway. For example, the HIV Vif protein achieves immune evasion by disrupting the IFN $\alpha$ mediated phosphorylation of STAT1 and STAT3 and reducing the expression of ISG15 (31). However, enterovirus 71 (EV71)

\section{REFERENCES}

1. Wen X, Zhu D, Cheng A, Wang M, Chen S, Jia R, et al. Molecular epidemiology of duck hepatitis a virus types 1 and 3 in China, 2010-2015. Transbound Emerg Dis. (2018) 65:10-5. doi: 10.1111/tbed.12741

2. Tsai H-J, Woolcock P. Viral infections of waterfowl. In: Swayne DE, Glisson JR, McDougald LR, Nolan LK, Suarez DL, Nair VL, editors. Diseases of Poultry. Vol. 13, Oxford, UK: John Wiley \& Sons, Inc. (2013). p. 417-63. inhibits the intracellular type I IFN signaling pathway by downregulating the expression of the JAK1 protein (40). We found that overexpression of SOCS3 significantly reduced the transcript levels of STAT1 and STAT3 and the antiviral proteins MX1 and OASL. However, when SOCS3 was co-transfected into cells with IFN $\alpha$, we found that the mRNA levels of STAT1 and STAT3 were not significantly reduced. We hypothesize that SOCS3 may inhibit the phosphorylation of STAT1 or STAT3, thereby affecting the expression of downstream MX1 and ultimately destroying the type I IFN signaling pathway.

In summary, the DHAV-1 CH60 strain inhibits the expression of IFN $\alpha$ by increasing the SOCS3 protein and SOCS3 then inhibits the mRNA expression of STAT1, STAT3 and the antiviral protein MX1, which ultimately promotes viral replication and thus indirectly assists in viral adaptation.

\section{ETHICS STATEMENT}

The study was approved by the Committee of Experiment Operational Guidelines and Animal Welfare of Sichuan Agricultural University (the approved permit number is XF2014-18). Experiments were conducted in accordance with approved guidelines.

\section{AUTHOR CONTRIBUTIONS}

JX conceived and carried out the experiments, analyzed the data, and wrote the manuscript. AC and MW conceived and supervised the study. X-XZ, ML, DZ, SC, RJ, YW, SZ, YL, YY, $\mathrm{LZ}, \mathrm{XC}$, and QY interpreted the data and revised the manuscript. All the authors reviewed the manuscript.

\section{FUNDING}

This work was supported by grants from National Natural Science Foundation of China (31472223), the China Agricultural Research System (CARS-42-17), the Sichuan Veterinary Medicine and Drug Innovation Group of the China Agricultural Research System (CARS-SVDIP) and the Special Fund for the Key Laboratory of Animal Disease and Human Health of Sichuan Province (2016JPT0004).

\section{SUPPLEMENTARY MATERIAL}

The Supplementary Material for this article can be found online at: https://www.frontiersin.org/articles/10.3389/fimmu. 2019.00731/full\#supplementary-material on the pathogenesis of DHV-1 in Pekin ducks and on the innate immune responses of ducks to infection. Arch Virol. (2014) 159:905-14. doi: 10.1007/s00705-013-1900-7

4. Ou X, Mao S, Cao J, Cheng A, Wang M, Zhu D, et al. Comparative analysis of virus-host interactions caused by a virulent and an attenuated duck hepatitis A virus genotype 1. PLOS ONE. (2017) 12:e0178993. doi: 10.1371/journal.pone.0178993 
5. Xie J, Wang M, Cheng A, Zhao XX, Liu M, Zhu D, et al. Cytokine storms are primarily responsible for the rapid death of ducklings infected with duck hepatitis A virus type 1. Sci Rep. (2018) 8:6596. doi: 10.1038/s41598-018-24729-w

6. Mao S, Ou X, Zhu D, Chen S, Ma G, Wang M, et al. Development and evaluation of indirect ELISAs for the detection of IgG, IgM and IgA1 against duck hepatitis A virus 1. J Virol Methods. (2016) 237:79-85. doi: 10.1016/j.jviromet.2016.08.019

7. Hu Q, Zhu D, Ma G, Cheng A, Wang M, Chen S, et al. A one-step duplex rRTPCR assay for the simultaneous detection of duck hepatitis A virus genotypes 1 and 3. J Virol Methods. (2016) 236:207-14. doi: 10.1016/j.jviromet.2016.07.011

8. Wen XJ. Detection, differentiation, and VP1 sequencing of duck hepatitis A virus type 1 and type 3 by a 1-step duplex reverse-transcription PCR assay. Poultry Sci. (2014) 93:2184-92. doi: 10.3382/ps.2014-04024

9. Shen Y, Cheng A, Wang M, Chen S, Jia R, Zhu D, et al. Development of an indirect ELISA method based on the VP3 protein of duck hepatitis A virus type 1 (DHAV-1) for dual detection of DHAV-1 and DHAV-3 antibodies. $J$ Virol Methods. (2015) 225:30-4. doi: 10.1016/j.jviromet.2015.08.016

10. Anchun C, Mingshu W, Hongyi X, Dekang Z, Xinran L, Haijuen C, et al. Development and application of a reverse transcriptase polymerase chain reaction to detect Chinese isolates of duck hepatitis virus type 1. J Microbiol Methods. (2009) 77:332-6. doi: 10.1016/j.mimet.2009.02.002

11. Yang $M$, Cheng A, Wang M, Xing H. Development and application of a onestep real-time Taqman RT-PCR assay for detection of Duck hepatitis virus type1. J Virol Methods. (2008) 153:55-60. doi: 10.1016/j.jviromet.2008.06.012

12. Cheng A, Liao D, Xie J, Chen X. Studies on duck viral hepatitis-pathogen isolation, identification and cultivation of attenuated viruses. Chin J Vet Med. (1993) 19:3-4.

13. Wang $M$, Cheng A, Chen X. Study on duck viral hepatitis-attenuated distribution and excretion in ducklings and adult ducks. J Prev Vet. (1997) 4:11-5.

14. Mao S, Wang M, Ou X, Sun D, Cheng A, Zhu D, et al. Virologic and immunologic characteristics in mature ducks with acute duck hepatitis A virus 1 infection. Front Immunol. (2017) 8:1574. doi: 10.3389/fimmu.2017.01574

15. Ou X, Wang $M$, Mao S, Cao J, Cheng $A$, Zhu D, et al. Incompatible translation drives a convergent evolution and viral attenuation during the development of live attenuated vaccine. Front Cell Infect Microbiol. (2018) 8:249. doi: 10.3389/fcimb.2018.00249

16. Xie J, Zeng Q, Wang M, Ou X, Ma Y, Cheng A, et al. Transcriptomic characterization of a chicken embryo model infected with duck hepatitis A virus Type 1. Front Immunol. (2018) 9:1845. doi: 10.3389/fimmu.2018.01845

17. Yang X, Cheng A, Wang M, Jia R, Sun K, Pan K, et al. Structures and corresponding functions of five types of Picornaviral 2A proteins. Front Microbiol. (2017) 8:1373. doi: 10.3389/fmicb.2017.01373

18. Zhang Y, Cao Q, Wang M, Jia R, Chen S, Zhu D, et al. The $3 \mathrm{D}$ protein of duck hepatitis A virus type 1 binds to a viral genomic $3^{\prime}$ UTR and shows RNA-dependent RNA polymerase activity. Virus Genes. (2017) 53:831-9. doi: 10.1007/s11262-017-1476-5

19. Cao J, Ou X, Zhu D, Ma G, Cheng A, Wang M, et al. The $2 \mathrm{~A} 2$ protein of duck hepatitis A virus type 1 induces apoptosis in primary cell culture. Virus Genes. (2016) 52:780-8. doi: 10.1007/s11262-016-1364-4

20. Sun D, Chen S, Cheng A, Wang M. Roles of the picornaviral 3C proteinase in the viral life cycle and host cells. Viruses. (2016) 8:82. doi: 10.3390/v8030082

21. Wen X, Cheng A, Wang M, Jia R, Zhu D, Chen S, et al. Recent advances from studies on the role of structural proteins in enterovirus infection. Future Microbiol. (2015) 10:1529-42. doi: 10.2217/fmb.15.62

22. Ou X, Mao S, Cao J, Ma Y, Ma G, Cheng A, et al. The neglected avian hepatotropic virus induces acute and chronic hepatitis in ducks: an alternative model for hepatology. Oncotarget. (2017) 8:81838-51. doi: 10.18632/oncotarget.19003

23. Ou X, Mao S, Jiang Y, Zhang S, Ke C, Ma G, et al. Viral-host interaction in kidney reveals strategies to escape host immunity and persistently shed virus to the urine. Oncotarget. (2017) 8:7336-49. doi: 10.18632/oncotarget.14227
24. Tamiya T, Kashiwagi I, Takahashi R, Yasukawa H, Yoshimura A. Suppressors of cytokine signaling (SOCS) proteins and JAK/STAT pathways: regulation of T-cell inflammation by SOCS1 and SOCS3. Arterioscler Thromb Vasc Biol. (2011) 31:980-5. doi: 10.1161/ATVBAHA.110.207464

25. Inagaki-Ohara $\mathrm{K}$, Kondo $\mathrm{T}$, Ito $\mathrm{M}$, Yoshimura A. SOCS, inflammation, and cancer. JAKSTAT. (2013) 2:e24053. doi: 10.4161/jkst.24053

26. Carow B, Rottenberg ME. SOCS3, a major regulator of infection and inflammation. Front Immunol. (2014) 5:58. doi: 10.3389/fimmu.2014.00058

27. Fu YZ, Su S, Gao YQ, Wang PP, Huang ZF, Hu MM, et al. Human cytomegalovirus tegument protein UL82 inhibits STING-mediated signaling to evade antiviral immunity. Cell Host Microbe. (2017) 21:231-43. doi: 10.1016/j.chom.2017.01.001

28. Sun D, Wang M, Wen X, Cheng A, Jia R, Sun K, et al. Cleavage of poly(A)binding protein by duck hepatitis A virus 3C protease. Sci Rep. (2017) 7:16261. doi: 10.1038/s41598-017-16484-1

29. Alexander WS. Suppressors of cytokine signalling (SOCS) in the immune system. Nat Rev Immunol. (2002) 2:410-6. doi: 10.1038/nri818

30. Mbow ML, Sarisky RT. What is disrupting IFN-alpha's antiviral activity? Trends Biotechnol. (2004) 22:395-9. doi: 10.1016/j.tibtech.2004.06.002

31. Gargan S, Ahmed S, Mahony R, Bannan C, Napoletano S, O’Farrelly C, et al. HIV-1 Promotes the degradation of components of the Type 1 IFN JAK/STAT pathway and blocks anti-viral ISG induction. EBioMed. (2018) 30:203-16. doi: 10.1016/j.ebiom.2018.03.006

32. Takeuchi O, Akira S. Innate immunity to virus infection. Immunol Rev. (2009) 227:75-86. doi: 10.1111/j.1600-065X.2008.00737.x

33. Akhtar LN, Benveniste EN. Viral exploitation of host SOCS protein functions. J Virol. (2011) 85:1912-21. doi: 10.1128/JVI.01857-10

34. Bode JG, Ludwig S, Ehrhardt C, Albrecht U, Erhardt A, Schaper F, et al. IFN-alpha antagonistic activity of $\mathrm{HCV}$ core protein involves induction of suppressor of cytokine signaling-3. FASEB J. (2003) 17:488-90. doi: 10.1096/fj.02-0664fje

35. Kim KA, Lin W, Tai AW, Shao RX, Weinberg E, De Sa Borges CB, et al. Hepatic SOCS3 expression is strongly associated with non-response to therapy and race in HCV and HCV/HIV infection. J Hepatol. (2009) 50:705-11. doi: 10.1016/j.jhep.2008.12.021

36. Bullen DV, Darwiche R, Metcalf D, Handman E, Alexander WS. Neutralization of interferon-gamma in neonatal SOCS1 ${ }^{-/-}$mice prevents fatty degeneration of the liver but not subsequent fatal inflammatory disease. Immunology. (2001) 104:92-8. doi: 10.1046/j.1365-2567.2001.01294.x

37. Liau NPD, Laktyushin A, Lucet IS, Murphy JM, Yao S, Whitlock E, et al. The molecular basis of JAK/STAT inhibition by SOCS1. Nat Commun. (2018) 9:1558. doi: 10.1038/s41467-018-04013-1

38. Kinjyo I, Hanada T, Inagaki-Ohara K, Mori H, Aki D, Ohishi M, et al. SOCS1/JAB is a negative regulator of LPS-induced macrophage activation. Immunity. (2002) 17:583-91. doi: 10.1016/S1074-7613(02)00446-6

39. Villarino A, Kanno Y, O'Shea J. Mechanisms and consequences of JakSTAT signaling in the immune system. Nat Immunol. (2017) 18:374-84. doi: 10.1038/ni.3691

40. Liu Y, Zhang Z, Zhao X, Yu R, Zhang X, Wu S, et al. Enterovirus 71 inhibits cellular type I interferon signaling by downregulating JAK1 protein expression. Viral Immunol. (2014) 27:267-76. doi: 10.1089/vim.2013.0127

Conflict of Interest Statement: The authors declare that the research was conducted in the absence of any commercial or financial relationships that could be construed as a potential conflict of interest.

Copyright (c) 2019 Xie, Wang, Cheng, Zhao, Liu, Zhu, Chen, Jia, Yang, Wu, Zhang, Liu, Yu, Zhang and Chen. This is an open-access article distributed under the terms of the Creative Commons Attribution License (CC BY). The use, distribution or reproduction in other forums is permitted, provided the original author(s) and the copyright owner(s) are credited and that the original publication in this journal is cited, in accordance with accepted academic practice. No use, distribution or reproduction is permitted which does not comply with these terms. 